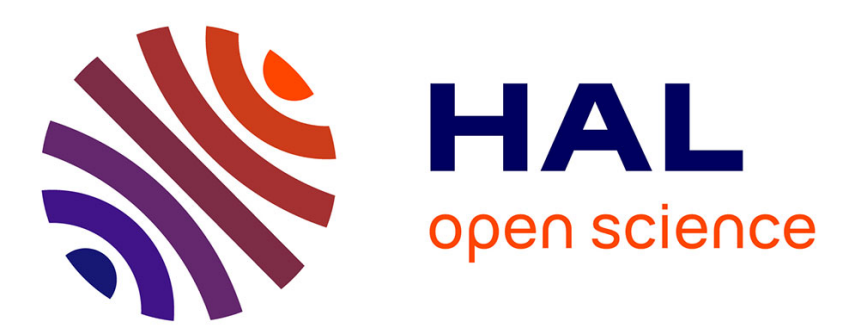

\title{
Head and neck target delineation using a novel PET automatic segmentation algorithm
}

B Berthon, M Evans, C Marshall, N Palaniappan, N Cole, V Jayaprakasam, T Rackley, E Spezi

\section{- To cite this version:}

B Berthon, M Evans, C Marshall, N Palaniappan, N Cole, et al.. Head and neck target delineation using a novel PET automatic segmentation algorithm. Radiotherapy \& Oncology, 2017, 122, pp.242 - 247. 10.1016/j.radonc.2016.12.008 . hal-03269455

\section{HAL Id: hal-03269455 https://hal.science/hal-03269455}

Submitted on 30 Jun 2021

HAL is a multi-disciplinary open access archive for the deposit and dissemination of scientific research documents, whether they are published or not. The documents may come from teaching and research institutions in France or abroad, or from public or private research centers.
L'archive ouverte pluridisciplinaire HAL, est destinée au dépôt et à la diffusion de documents scientifiques de niveau recherche, publiés ou non, émanant des établissements d'enseignement et de recherche français ou étrangers, des laboratoires publics ou privés. 


\title{
Head and neck target delineation using a novel PET automatic segmentation algorithm
}

\author{
B. Berthon ${ }^{\mathrm{a}, *, 1}$, M. Evans ${ }^{\mathrm{b}}$, C. Marshall ${ }^{\mathrm{a}}$, N. Palaniappan ${ }^{\mathrm{b}}$, N. Cole ${ }^{\mathrm{b}}$, V. Jayaprakasam $^{\mathrm{b}}$, T. Rackley $^{\mathrm{b}}$, \\ E. Spezi ${ }^{\text {b,c }}$
}

${ }^{\mathrm{a}}$ Wales Research Er Diagnostic PET Imaging Centre; ${ }^{\mathrm{b}}$ Velindre Cancer Centre; and ${ }^{\mathrm{c}}$ School of Engineering, Cardiff University, Cardiff, UK

\section{A R T I C L E I N F O}

\section{Article history:}

Received 24 June 2016

Received in revised form 5 December 2016

Accepted 5 December 2016

Available online $\mathrm{xxxx}$

\section{Keywords:}

Positron Emission Tomography

Image Segmentation

Intensity Modulated Radiation Therapy

Automatic PET segmentation

\begin{abstract}
A B S T R A C T
Purpose: To evaluate the feasibility and impact of using a novel advanced PET auto-segmentation method in Head and Neck (H\&N) radiotherapy treatment (RT) planning.

Methods: ATLAAS, Automatic decision Tree-based Learning Algorithm for Advanced Segmentation, previously developed and validated on pre-clinical data, was applied to ${ }^{18} \mathrm{~F}$-FDG-PET/CT scans of $20 \mathrm{H} \& \mathrm{~N}$ patients undergoing Intensity Modulated Radiation Therapy. Primary Gross Tumour Volumes (GTVs) manually delineated on $\mathrm{CT} / \mathrm{MRI}$ scans (GTVP $\mathrm{P}_{\mathrm{CT} / \mathrm{MRI}}$ ), together with ATLAAS-generated contours (GTV $p_{\text {ATLAAS }}$ ) were used to derive the RT planning GTV (GTV $\left.p_{\text {final }}\right)$. ATLAAS outlines were compared to CT/MRI and final GTVs qualitatively and quantitatively using a conformity metric.

Results: The ATLAAS contours were found to be reliable and useful. The volume of GTVp $\mathrm{p}_{\text {ATLAAS }}$ was smaller than GTV $\mathrm{p}_{\mathrm{CT} / \mathrm{MRI}}$ in $70 \%$ of the cases, with an average conformity index of 0.70 . The information provided by ATLAAS was used to grow the GTV $\mathrm{p}_{\mathrm{CT} / \mathrm{MRI}}$ in 10 cases (up to $10.6 \mathrm{~mL}$ ) and to shrink the $\mathrm{GTV}_{\mathrm{CT} / \mathrm{MRI}}$ in 7 cases (up to $12.3 \mathrm{~mL}$ ). ATLAAS provided complementary information to $\mathrm{CT} / \mathrm{MRI}$ and $\mathrm{GTV}_{\text {ATLAAS }}$ contributed to up to $33 \%$ of the final GTV volume across the patient cohort.

Conclusions: ATLAAS can deliver operator independent PET segmentation to augment clinical outlining using CT and MRI and could have utility in future clinical studies.
\end{abstract}

(c) 2017 Elsevier B.V. All rights reserved. Radiotherapy and Oncology xxx (2017) xxx-xxx
Positron Emission Tomography (PET) imaging using 18FFluorodeoxyglucose (FDG) plays an increasingly valuable role in Radiotherapy Treatment (RT) planning for a number of cancers [1]. Loco-regional recurrences have been shown to occur in PETavid regions of the tumour [2] and studies have demonstrated the feasibility and usefulness of PET/CT-guided Intensity Modulated Radiation Therapy (IMRT) planning [3]. PET/CT-based outlining can lead to more accurate and reproducible delineation of the Gross Tumour Volume (GTV), compared to outlining done using CT alone [4]. The PET-based GTV is usually smaller than the CT based volume [5,6]. Nishioka et al. showed with 21 oropharyngeal and nasopharyngeal cancer patients that adjacent normal tissue, particularly parotids, could be spared in $71 \%$ of patients when using PET in the delineation process [7], which could potentially lead to reduced long term morbidity, xerostomia and improved quality of life.

\footnotetext{
* Corresponding author at: Institut Langevin, 17 rue Moreau, 75012 Paris, France. E-mail address: beatrice.walker@espci.fr (B. Berthon).

1 Dr. Berthon is now at Institut Langevin, ESPCI Paris, PSL Research University, CNRS UMR 7587, INSERM U979.
}

Although FDG-PET has been adopted in oncology as a key tool in diagnostic imaging, its use in RT planning has, until now, been limited due to a lack of consensus on GTV delineation method. The low resolution of PET coupled with the proximity to the tumour of other metabolically active structures make the delineation challenging. In particular in the Head and Neck (H\&N), organs such as the pharyngeal muscles, spinal cord and salivary glands, which should be spared to minimise morbidity and improve quality of life, can generate additional background FDG uptake.

Manual PET-based GTV delineation, currently used in most centres, is time consuming and highly operator-dependent and several studies have shown significant variations in the GTV delineated by different operators using PET $[5,8]$. This has led to the development and recommended use of various PET automatic segmentation (PET-AS) methods for H\&N [9]. However, only a small number of prospective clinical studies have reported on the use of PET-AS in RT planning [6]. Comparing different studies is difficult because of the different PET-AS methods used. Basic thresholding methods lack accuracy and reliability [10,11], but more advanced PET-AS methods, such as gradient-based, clustering or region-growing approaches are rarely used, and their impact on RT planning is still 


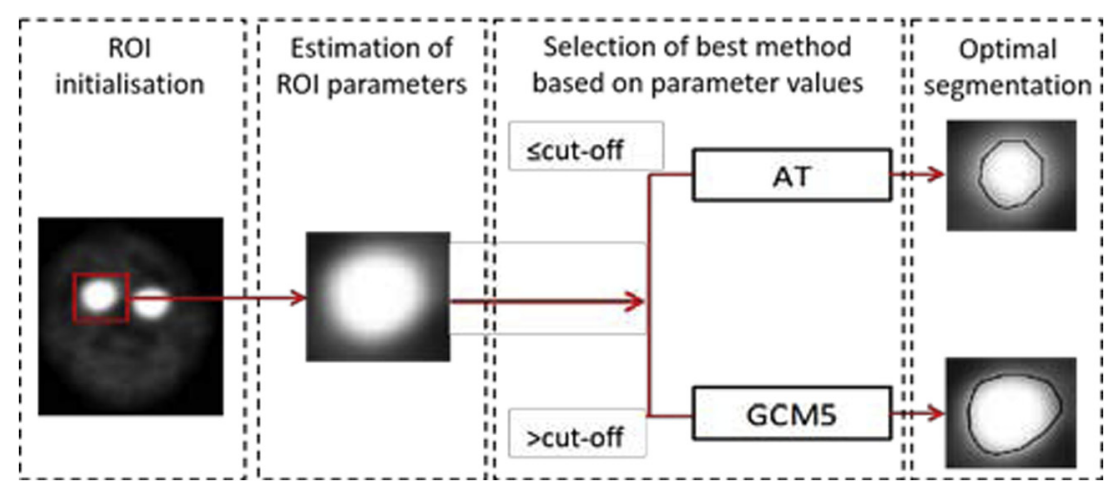

Fig. 1. Example of steps in the decision tree method implemented in the ATLAAS segmentation model.

unclear. There is a need for studies investigating the feasibility and clinical benefits of using advanced PET-AS in RT planning.

This prospective study investigated the use of an optimised PET-AS tool, developed and validated in house using phantom and clinical PET data [12,13], for GTV delineation in the RT planning of 20 oropharyngeal cancer patients. We evaluated the feasibility and impact of including this method into the RT planning process.

\section{Methods}

\section{The ATLAAS optimised segmentation model}

PET-AS was performed using the Automatic decision Tree-based Learning Algorithm for Advanced Segmentation (ATLAAS) ${ }^{2}$ method developed at our centre. The ATLAAS model is designed to select the most accurate PET-AS method for a given PET image. This is achieved using a decision tree supervised machine learning method, optimised with a training dataset for which the segmentation outcome is known, to achieve optimal performance for cases in which the outcome is not known. ATLAAS is described elsewhere [14], and its accuracy was shown for 6 classes of advanced PET-AS methods used to segment a large range of data including simulated H\&N tumours, and phantom $\mathrm{H} \& \mathrm{~N}$ images of complex and realistic tumours obtained with a sub-resolution printed sandwich phantom [15]. ATLAAS was optimised for H\&N data using 65 sub-resolution printed sandwich phantom images. The optimised version included the two algorithms Adaptive Thresholding method (AT) and Gaussian mixture models Clustering Method using 5 clusters (GCM5), described in previous work [12]. The best method was predicted on the basis of $\mathrm{TBR}_{\text {peak }}$ defined as the ratio between the tumour peak intensity value, (mean value in a $1 \mathrm{~cm}^{3}$ sphere centred on the maximum intensity voxel) and the background intensity (mean intensity in a $1 \mathrm{~cm}$ thick extension of a thresholded volume at $50 \%$ of the peak intensity value). An example of the typical steps involved in the segmentation with ATLAAS is given in Fig. 1. The ATLAAS model was implemented for this work in the Computational Environment for Radiotherapy Research (CERR) [16]. The segmentation accuracy was evaluated by quantifying the overlap between the segmented and true contour using the Dice Similarity Coefficient (DSC) described in other work [17].

\section{Acquisition of clinical data}

The POSITIVE (Optimization of Positron Emission Tomography based Target Volume Delineation in Head and Neck Radiotherapy) study was set up to test ATLAAS for the first time in patients under-

\footnotetext{
2 Patent pending No PCT/GB2015/052981.
}

going H\&N radiotherapy (REC No. 12/WA/0083) and was carried out at Velindre Cancer Centre (UK). Twenty stage III/IVa-b oropharyngeal cancer patients were recruited after informed consent to the study. The patients were treated with neoadjuvant (induction) chemotherapy followed by radical chemoradiotherapy (66 Gy in 30 fractions over 6 weeks) using IMRT. A planning FDG PET/CT scan was carried out on a GE Discovery 690 PET/CT scanner before chemotherapy to avoid changes in tumour volumes prior to outlining. The scans were acquired 90 min after FDG administration in the treatment position with an RT immobilisation shell. The PET was acquired using 6-8 bed positions of 3 min each. The patient was injected with contrast for a subsequent CT used in the planning process. The images were reconstructed to $512 \times 512$ voxels for CT and $256 \times 256$ voxels for PET, using the algorithm Vue Point FX (24 subsets, 2 iterations, $6.4 \mathrm{~mm}$ cut-off) including CT-based attenuation-, scatter- and Time-Of-Flight corrections.

Six weeks on average separated the PET/CT planning scan and the start of RT. The fit of the immobilisation shells was adjusted if needed after induction chemotherapy and the patient was reoutlined and re-planned if necessary using the original CT/MRI/ PET scan. Reporting was done by PET specialist radiologists after acquisition of the planning scans.

\section{Workflow and analysis}

MRI scans acquired before recruitment were available for all patients and were fused to the planning PET-CT scan using the Mutual Information registration algorithm in the ProSoma software (MedCom GmbG, Darmstadt, Germany).

Planning scans for the first 10 patients recruited were used to validate the workflow and verify that ATLAAS provided relevant contours for use. In this subgroup the primary GTVs were manually outlined by three consultant radiation oncologists, in discussion with a specialist PET radiologist, on the registered PET/CT, using the software VelocityAI (Varian Medical Systems, Palo Alto, USA). The resulting GTV contours in terms of their volume and geometrical overlap, using the DSC index.

Once the ATLAAS output was verified, another 10 oropharyngeal cancer patients were recruited for the study. Manual delineation of the primary GTV was performed by the consultant radiation oncologists on the fused MRI and CT images in ProSoma (GTV $\mathrm{p}_{\mathrm{CT} / \mathrm{MRI}}$ ). ATLAAS (GTV $\mathrm{p}_{\text {ATLAAS }}$ ) contours were then imported into ProSoma where the final GTV $\left(G_{T V} p_{\text {final }}\right)$ was drawn by the treating clinician using all the available contour data.

The use of the additional information brought by ATLAAS contours was evaluated by comparing the different contours $\left(G T V p_{\text {CT/MRI }}, G T V p_{\text {ATLAAS, }}\right.$ and GTVp $\left.p_{\text {final }}\right)$ for each patient, in terms of volume and geometrical overlap using the DSC. In addition, 
Table 1

GTVp volumes and DSC index for manual and ATLAAS contours.

\begin{tabular}{|c|c|c|c|c|c|c|c|c|c|c|c|}
\hline & \multicolumn{10}{|c|}{ Patient no } & \multirow[t]{2}{*}{ Mean } \\
\hline & 11 & 12 & 13 & 14 & 15 & 16 & 17 & 18 & 19 & 20 & \\
\hline Final volume $(\mathrm{mL})$ & 33.1 & 45.9 & 21.5 & 38.2 & 27.5 & 54.7 & 33.1 & 19.0 & 33.8 & 17.4 & - \\
\hline $\mathrm{CT} / \mathrm{MRI}$ volume $(\mathrm{mL})$ & 27.1 & 40.6 & 19.8 & 32.8 & 26.9 & 60.8 & 28.5 & 16.6 & 31.3 & 15.6 & - \\
\hline ATLAAS volume (mL) & 27.3 & 41.9 & 7.8 & 26.2 & 15.6 & 52.5 & 29.0 & 16.1 & 23.7 & 8.6 & - \\
\hline DSC(ATLAAS vs CT/MRI) & 0.77 & 0.76 & 0.44 & 0.75 & 0.67 & 0.82 & 0.74 & 0.74 & 0.76 & 0.51 & 0.70 \\
\hline DSC(ATLAAS vs final) & 0.90 & 0.77 & 0.53 & 0.81 & 0.68 & 0.97 & 0.84 & 0.92 & 0.83 & 0.58 & 0.77 \\
\hline
\end{tabular}

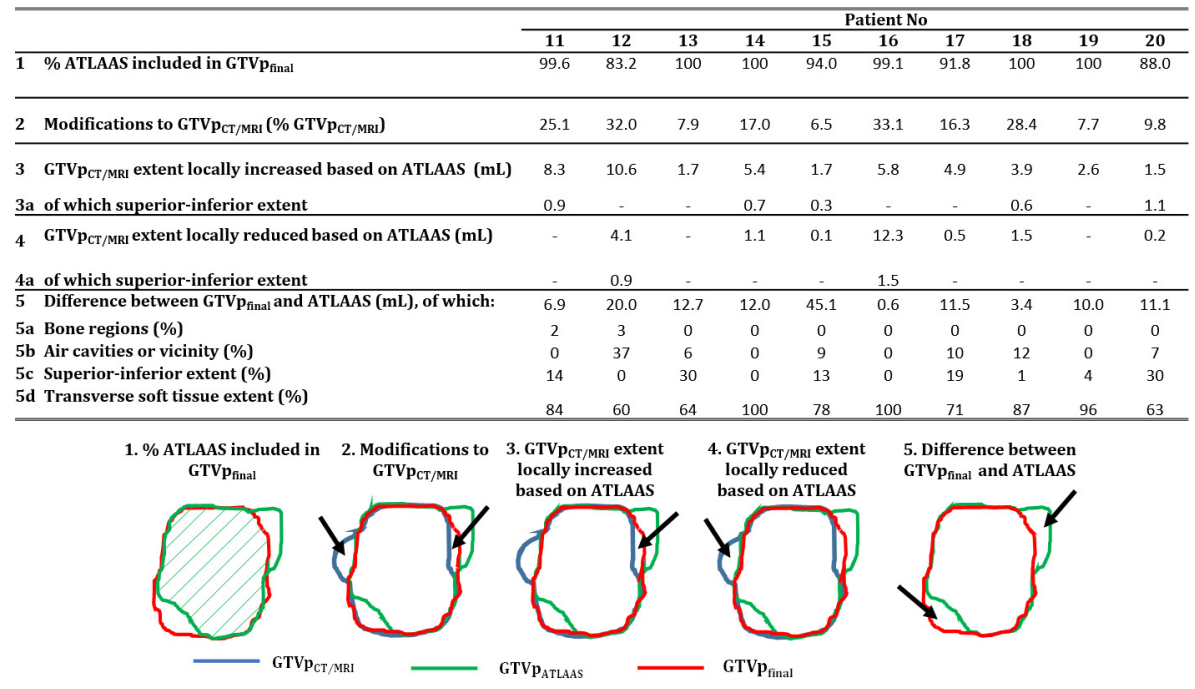

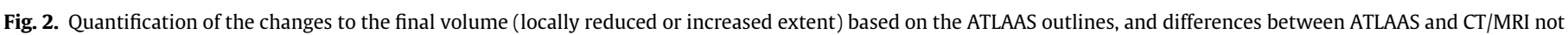
taken into account in the final GTV. Calculations corresponding to the different rows are schematically described under the table.

the clinicians were asked to report any changes made to the $G T V p_{\text {final }}$ due to the ATLAAS contour. Lymph nodes, which are well defined on CT/MRI images, were not outlined using ATLAAS, and are therefore not reported on in this paper.

\section{Results}

The patient cohort included 17 men and 3 women with a median age of 63 years. Ten patients had tonsillar tumours, 8 base of tongue tumours and 2 soft palate tumours. Two patients needed re-planning after induction chemotherapy.

In the preliminary group of 10 patients, ATLAAS successfully delineated the PET-avid tumour for all patients. The segmentation of the tumour ROI was fully automatic and took no more than $2 \mathrm{~min}$ on a dual core $3.1 \mathrm{GHz}$ processor. GTVpatLAAS were smaller than the manually delineated GTVP The mean DSC between GTV 0.7 is considered to be an indicator of good overlap [18]. On the basis of these results, it was decided that only ATLAAS and $\mathrm{CT} / \mathrm{MRI}$ contours would be used for the subsequent 10 patients recruited.

A comparison in terms of volume and conformity between the GTVp delineated using ATLAAS and both CT/MRI-based and final contours delineated by the investigators is presented in Table 1. ATLAAS volumes were smaller than the corresponding CT/MRI volumes in 7 out of 10 cases, and were within $10 \%$ of CT/MRI volumes in 4 out of 10 cases. The spatial conformity of GTVp ATLAAS $_{\text {and }}$ $\mathrm{GTV}_{\mathrm{CT} / \mathrm{MRI}}$ was 0.70 DSC on average. GTV $\mathrm{p}_{\text {ATLAAS }}$ and GTVp $\mathrm{p}_{\text {final }}$ were close, with neither being larger than $30 \%$ of the other, in 6 out of the 10 cases. GTV $p_{\text {final }}$ volumes were larger than the
GTVp $\mathrm{p}_{\text {ATLAAS }}$ in all cases. However, the ATLAAS volumes showed good conformity to the final contour, with an average DSC of 0.77 .

Fig. 2 reports the details of the global and local changes to the final volume based on ATLAAS, and outlines the differences between ATLAAS and CT/MRI contours not taken into account in the final GTV. For instance, the data in the top row of the table shows that more than $83 \%$ of the ATLAAS volume was included in the final GTV for all patients, and $100 \%$ of the ATLAAS volume was included in the final GTV in 4 cases. The second row reports the proportion of the CT/MRI volume modified on the basis of the ATLAAS outline. This value ranged from $6.5 \%$ to $33 \%$. This modification could include both additional extension of the volume when the ATLAAS contour was outside the GTV $\mathrm{p}_{\mathrm{CT} / \mathrm{MRI}}$ or local reduction of the volume in cases where the inverse was true. This is detailed in rows 3-5 as illustrated under the table.

Fig. 3 illustrates specific differences found between GTV $\mathrm{p}_{\mathrm{CT} / \mathrm{MRI}}$, $G T V p_{\text {ATLAAS }}$ and $G T V p_{\text {final }}$ overlaid on the corresponding CT/PET scan, for seven clinical cases of interest.

\section{Extending The GTV locally based on ATLAAS}

As reported in the third row of Fig. 2, GTV $\mathrm{p}_{\mathrm{CT} / \mathrm{MRI}}$ was locally extended based on the information provided by ATLAAS (cf. Fig. 3a) for all clinical cases, with up to $10 \mathrm{~mL}$ added to make the final volume. Visual examination and reporting by the clinicians showed that this was done when additional disease extension was detected by ATLAAS, and confirmed by clinical or CT/MRI findings. This included larger superior-inferior disease extension (by up to $1.1 \mathrm{~cm}$ in one patient as reported in Fig. $3 \mathrm{~b}$ ), and disease extension identified across the midline (cf. Fig. 3c). 


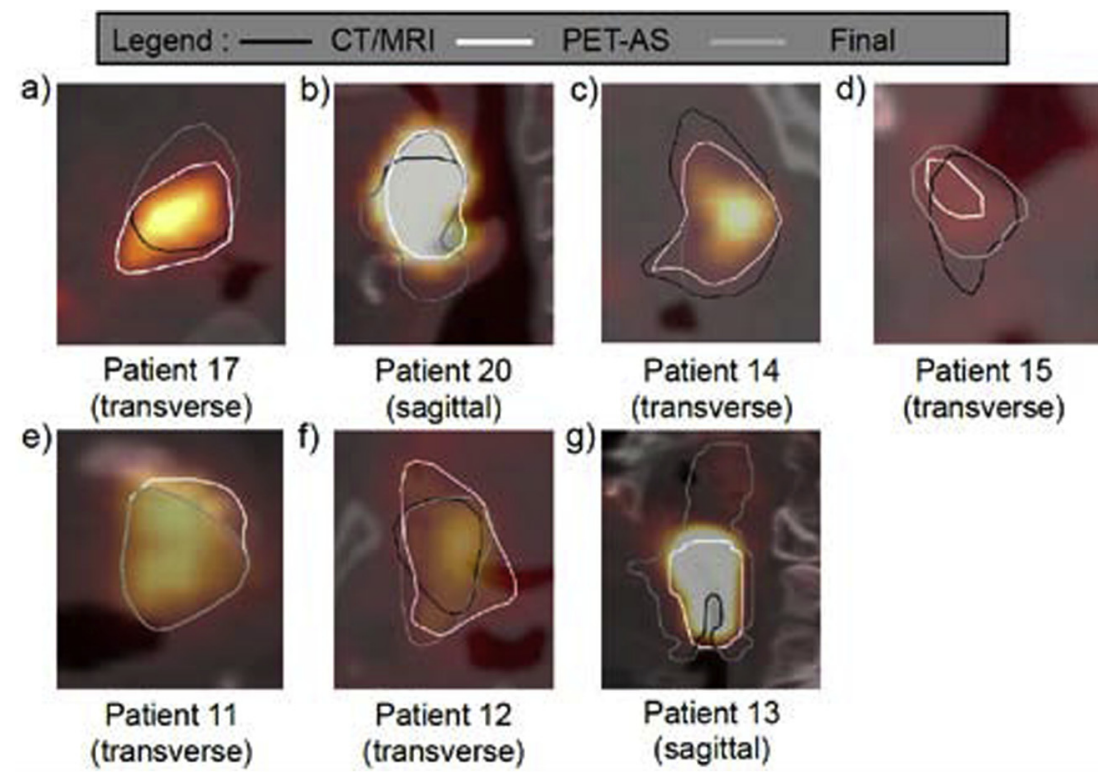

Fig. 3. GTV $\mathrm{GT}_{\mathrm{C} / \mathrm{MRI}}, \mathrm{GTV} \mathrm{p}_{\mathrm{AtLAAS}}$, and $\mathrm{GTV} \mathrm{p}_{\text {final }}$ compared for 7 clinical cases.

\section{Reducing the GTV locally based on ATLAAS}

As shown in the fourth row of Fig. 2, local reduction (on one or more transverse slices) of the CT/MRI volume based on ATLAAS was observed for 7 patients. For two patients, it resulted in a reduction to the final volume of more than $2 \mathrm{~mL}$. The extent of the contours was locally reduced when the smaller disease extent indicated by ATLAAS was in agreement with the clinical findings and the CT or the MRI information. The extent was also reduced in the superior-inferior direction for two patients (by $1.5 \mathrm{~cm}$ for patient No 16). In cases of largely conflicting information between image modalities, the CT/MRI contour was reduced down to a compromise following the edge of the anatomical structures, as depicted in Fig. 3d.

\section{ATLAAS information discarded}

Differences between GTV $\mathrm{p}_{\mathrm{CT} / \mathrm{MRI}}$ and GTV $\mathrm{p}_{\text {ATLAAS }}$ were not considered in the final GTV when they included:

(a) bone $(0.1 \mathrm{~mL}$ for patient No 11 , cf. Fig. 3e),

(b) air (for 5 patients, up to $6.6 \mathrm{~mL}$ for patient No 12, cf. Fig. 3f),

(c) different superior-inferior disease extension in GTV $p_{\text {ATLAAS }}$ which was not confirmed by anatomical imaging or clinical examinations (for 6 patients, up to $6.4 \mathrm{~mL}$ for patient No 13, cf. Fig. 3g),

(d) different transverse disease extension unconfirmed by anatomical imaging or clinical examinations (cf. some regions in Fig. 3f).

In these cases, the difference between GTVp $p_{\text {ATLAAS }}$ and GTV $p_{\text {final }}$ (expressed in $\mathrm{mL}$ ), is given in row 5 of Fig. 2 and includes both over and under contouring.

\section{Discussion}

In this study, we investigated the clinical feasibility of using the novel ATLAAS optimised segmentation model in $20 \mathrm{H} \& \mathrm{~N}$ cancer patients undergoing radical chemoradiotherapy. ATLAAS was applied for the first time to 20 prospectively recruited patients in a clinical trial with a strict scanning protocol, which involved expert PET radiologist and $\mathrm{H} \& \mathrm{~N}$ radiation oncologists. It was prospectively used, in combination with manual CT/MRI data, to derive the final GTV for use in RT planning. To the best of our knowledge, advanced PET-AS methods (beyond simple thresholding) have only been included as part of RT treatment planning in two studies in H\&N cancer $[19,20]$, which were based on the same gradient-based segmentation method. In this work, we additionally evaluated the impact of using the PET-AS contour on local modifications of the final GTV contour.

ATLAAS had previously shown accuracy and robustness on phantom and simulated data for the evaluation of H\&N PET scans [14]. Evaluation on images from the 10 first patients involved in this study showed that ATLAAS provided PET-avid GTVs for all patients with a high degree of similarity to PET GTVs manually delineated by experts. In addition, the segmentation was fully automatic and therefore reproducible, and lasted no more than 2 min per patient. The use of ATLAAS instead of manual PET/CT outlining for the 10 subsequent patients in this study, considerably reduced the clinicians' workload and removed inter-observer variability. We have shown that ATLAAS not only could segment the PET-avid areas of disease reliably in patients compared to manual PET outlining but that it could also add valuable information to guide clinical delineation of the primary GTV.

The ATLAAS contours were smaller than the CT/MRI contours in most cases, which is in agreement with findings from other studies where threshold-based delineation was used for H\&N patients [21]. Furthermore, the ATLAAS derived contours provided additional information to anatomical contours manually drawn on CT and MRI. This is in line with the study by Newbold et al. in 19 $\mathrm{H} \& \mathrm{~N}$ patients, where threshold-based delineation was used to derive the PET-based GTV [22]. In our study, we found that additional information from ATLAAS included (a) identification of superior-inferior disease extension, and extension across the midline not seen on CT (e.g. Fig. 3c), and (b) other disease extension boundaries differing from anatomical data. The information provided by ATLAAS was used in all patients and this shows the confidence of the clinicians in the usefulness of our segmentation method for RT planning at our centre. The clinician's judgment and expertise and the additional clinical data available (endoscopy or clinical examination results) remained paramount in the process. Nevertheless ATLAAS was very useful (a) in confirming the GTV outline when this was close to the CT/MRI based contour, and (b) as a delineation guide when in disagreement with $\mathrm{CT} /$ 
MRI based contours, due for instance to different patient positioning and/or poor image registration.

We have methodically investigated the impact of ATLAAS on the final GTV for our cohort. We found that although ATLAAS led to reducing the extension in some areas of the $\mathrm{GTV}_{\mathrm{CT} / \mathrm{MRI}}$ for 7 patients, the PET information led to a globally smaller final GTV for only 1 patient. This is in line with the findings of Ciernik et al. for a cohort of $12 \mathrm{H} \& \mathrm{~N}$ patients [5], and Paulino et al. for $40 \mathrm{H} \& \mathrm{~N}$ patients [23], both using manual PET segmentation. This confirms the suggestion that clinicians may not be prepared yet to reduce the GTV volume based on PET. Indeed, although some studies have shown that PET-AS contours can accurately identify the whole tumour burden in laryngeal cancer [4,24], it may be more useful for defining the metabolically active tumour region, especially for tumours which can be highly heterogeneous such as in the H\&N [25]. This is in line with the suggestion of considering the Biological Tumor Volume as defined by Ling et al. [26], which can be used for dose escalation $[27,28]$ to increase the dose to the biologically active tumour while sparing the surrounding tissue. The ATLAAS model could be useful for determining, with a consistent and operator independent approach, highly metabolically active areas of the tumour requiring a radiation boost and it could therefore be extremely useful for treatment plan adaptation. Correlation with additional information and clinical input would still be required in finalising the volumes for dose escalation.

Differences between the GTVp final and GTVpatLAAs volumes were in the range $[0.6,45] \mathrm{mL}$ (cf. row 5 of Fig. 2). CT/MRI based outlining was preferred when: (a) no PET signal was found in abnormal mucosa (Fig. 3g), (b) high PET uptake was observed in and around air cavities and/or bone (Fig. $3 \mathrm{c}$ and $\mathrm{h}$ ) due to signal spill-out or inflammation. Spill-out effects can be corrected with CT-based thresholding, whereas unconfirmed soft tissue extensions of the disease, which represent a large part of the differences observed between CT/MRI and PET contours ( $\mathrm{cf}$. rows $5 \mathrm{c}$ and $5 \mathrm{~d}$ of Fig. 2), are inherent to the difference between modalities.

One of the limitations of this study is that we could not carry out a full comparison between GTVp ATLAAS $_{\text {and }}$ ane PET GTV outlined manually without reference to anatomical data from the CT scan. In this case the correlation between manual and GTVp $p_{\text {ATLAAS }}$ could have been greater because based on the same underlying data. In addition, this work was carried out as a single centre study. Both limitations shall be addressed in the design of a forthcoming multicentre clinical trial.

\section{Conclusions}

The ATLAAS optimised segmentation model based on the decision tree machine learning method is a novel, fast and operator independent tool for tumour delineation in radiotherapy treatment planning of Head and Neck cancer. ATLAAS can potentially be applied to any tumour site and tumour type and holds promise for future multi-centre clinical studies investigating the use of PET in radiotherapy outlining, prior to starting treatment and also for adaptive re-planning of residual metabolically active disease during treatment.

\section{Conflict of interest}

The authors declare that they have no conflict of interest.

\section{Acknowledgements}

This work was funded by Cancer Research Wales grants No. 7061 and 2476 . The funder was not involved in the study design, conception, data collection acquisition and analysis nor writing or submission of this manuscript.

\section{References}

[1] MacManus M, Nestle U, Rosenzweig KE, Carrio I, Messa C, Belohlavek O, et al. Use of PET and PET/CT for radiation therapy planning: IAEA expert report 2006-2007. Radiother Oncol 2009:91:85-94.

[2] Due AK, Vogelius IR, Aznar MC, Bentzen SM, Berthelsen AK, Korreman SS, et al. Recurrences after intensity modulated radiotherapy for head and neck squamous cell carcinoma more likely to originate from regions with high baseline [18F]-FDG uptake. Radiother Oncol 2014:111:360-5.

[3] Wang D, Schultz C, Jursinic P. Initial experience of FDG-PET/CT guided IMRT of Head and Neck carcinoma. Int J Radiat Oncol Biol Phys 2006;65:143-51.

[4] Daisne J-F, Duprez T, Weynand B, Lonneux M, Hamoir M, Reychler H, et al. Tumor volume in pharyngolaryngeal squamous cell carcinoma: comparison at CT, MR imaging, and FDG PET and validation with surgical specimen. Radiology 2004;233:93-100.

[5] Ciernik I, Dizendorf E, Baumert B, Reiner B, Burger C, Davis J, et al. Radiation treatment planning with an integrated positron emission and computer tomography (PET/CT): a feasibility study. Int J Radiat Oncol Biol Phys 2003:57:853-63.

[6] Deantonio L, Beldì D, Gambaro G, Loi G, Brambilla M, Inglese E, et al. FDG-PET/ $\mathrm{CT}$ imaging for staging and radiotherapy treatment planning of head and neck carcinoma. Radiat Oncol 2008;3:29.

[7] Nishioka T, Shiga T, Shirato H, Tsukamoto E, Tsuchiya K, Kato T, et al. Image fusion between 18 FDG-PET and MRI/CT for radiotherapy planning of oropharyngeal and nasopharyngeal carcinomas. Int J Radiat Oncol Biol Phys 2002:53:1051-7.

[8] Caldwell CB, Mah K, Ung YC, Danjoux CE, Balogh JM, Ganguli SN, et al. Observer variation in contouring gross tumor volume in patients with poorly defined non-small-cell lung tumors on CT: the impact of 18FDG-hybrid PET fusion. Int J Radiat Oncol Biol Phys 2001;51:923-31.

[9] Grégoire V, Jeraj R, Lee JA, O'Sullivan B. Radiotherapy for head and neck tumours in 2012 and beyond: conformal, tailored, and adaptive? Lancet Oncol 2012:13:e292-300.

[10] Nestle U, Kremp S, Schaefer-Schuler A, Sebastian-Welsch C, Hellwig D, Ru C. Comparison of different methods for delineation of 18F-FDG PET-positive tissue for target volume definition in radiotherapy of patients with non-small cell lung cancer. J Nucl Med 2005;46:1342-8.

[11] Ford EC, Kinahan PE, Hanlon L, Alessio A, Rajendran J, Schwartz DL, et al. Tumor delineation using PET in head and neck cancers: threshold contouring and lesion volumes. Med Phys 2006:33:4280-8.

[12] Berthon B, Marshall C, Edwards A, Evans M, Spezi E. Influence of cold walls on PET image quantification and volume segmentation. Med Phys 2013;40:1-13.

[13] Berthon B, Marshall C, Evans M, Spezi E. Evaluation of advanced automatic PET segmentation methods using non-spherical thin-wall inserts. Med Phys 2014;41:22502.

[14] Berthon B, Marshall C, Evans M, Spezi E. ATLAAS: an Automatic decision treebased learning algorithm for advanced segmentation. Phys Med Biol 2016;61:4855-69.

[15] Berthon B, Marshall C, Holmes RB, Spezi E. A novel phantom technique for evaluating the performance of PET auto-segmentation methods in delineating heterogeneous and irregular lesions. Eur J Nucl Med Mol Imaging Phys 2015;2.

[16] Deasy JO, Blanco AI, Clark VH. CERR: a computational environment for radiotherapy research. Med Phys 2003;30:979-85.

[17] Dice L. Measures of the amount of ecologic association between species. Ecology 1945;26:297-302.

[18] Zou KH, Warfield SK, Bharatha A, Tempany CMC, Kaus MR, Haker SJ, et al. Statistical validation of image segmentation quality based on a spatial overlap index. Acad Radiol 2004;11:178-89.

[19] Geets X, Tomsej M, Lee JA, Duprez T, Coche E, Cosnard G. Adaptive biological image-guided IMRT with anatomic and functional imaging in pharyngolaryngeal tumors: impact on target volume delineation and dose distribution using helical tomotherapy. Radiother Oncol 2007;85:105-15.

[20] Leclerc M, Lartigau E, Lacornerie T, Daisne J, Kramar A, Grégoire V. Primary tumor delineation based on 18FDG PET for locally advanced head and neck cancer treated by chemo-radiotherapy. Radiother Oncol 2015;116:87-93.

[21] Greco C, Nehmeh SA, Schoder H, Gonen L, Raphael B, Stambuk HE, et al. Evaluation of different methods of 18F-FDG-PET target volume delineation in the radiotherapy of head and neck cancer. Am J Clin Oncol 2008:31:439-45.

[22] Newbold KL, Partridge M, Cook G, Sharma B, Rhys-Evans P, Harrington KJ, et al. Evaluation of the role of 18FDG-PET/CT in radiotherapy target definition in patients with head and neck cancer. Acta Oncol 2008;47:1229-36.

[23] Paulino AC, Koshy M, Howell R, Schuster D, Davis LW. Comparison of CT- and FDG-PET-defined gross tumor volume in intensity-modulated radiotherapy for head-and-neck cancer. Int J Radiat Oncol Biol Phys 2005;61:1385-92.

[24] Geets X, Lee JA, Bol A, Lonneux M, Grégoire V. A gradient-based method for segmenting FDG-PET images: methodology and validation. Eur J Nucl Med Mol Imaging 2007;34:1427-38.

[25] Hoogsteen I, Marres H, Bussink J, van der Kogel A, Kaanders J. Tumor microenvironment in head and neck squamous cell carcinomas: predictive value and clinical relevance of hypoxic markers. A review. Head Neck 2007;29:591-604. 
[26] Ling CC, Humm J, Larson S, Amols H, Fuks Z, Leibel S, et al. Towards multidimensional radiotherapy (MD-CRT): biological imaging and biological conformality. Int J Radiat Oncol Biol Phys 2000;47:551-60.

[27] van Elmpt W, De Ruysscher D, van der Salm A, Lakeman A, van der Stoep J, Emans D, et al. The PET-boost randomised phase II dose-escalation trial in nonsmall cell lung cancer. Radiother Oncol 2012;104:67-71.
[28] Even AJG, van der Stoep J, Zegers CML, Reymen B, Troost EGC, Lambin P, et al. PET-based dose painting in non-small cell lung cancer: comparing uniform dose escalation with boosting hypoxic and metabolically active sub-volumes. Radiother Oncol 2015;116. 